\title{
Physico-chimie et qualité des eaux des forages d'hydraulique villageoise dans la région centrale au Togo
}

\author{
Akpénè Amenuvevega DOUGNA ${ }^{1}$, Masamaéya Dadja-Toyo GNAZOU ${ }^{1,2 *}$, \\ Tomkouani KODOM ${ }^{1}$, Gbandi DJANEYE-BOUNDJOU ${ }^{1}$ et Moctar L. BAWA ${ }^{1}$ \\ ${ }^{1}$ Laboratoire de Chimie des Eaux, Faculté des Sciences, Université de Lomé, B.P. 1515, Lomé, Togo. \\ ${ }^{2}$ Département de Géologie, Faculté Des Sciences, Université de Lomé, B.P. 1515, Lomé, Togo. \\ *Auteur correspondant, E-mail : adougna@yahoo.fr ; Tél. (00228) 90964323
}

\section{RESUME}

Cette étude a porté sur la caractérisation physico-chimique des eaux de forages d'hydraulique villageoise des aquifères du socle de la région centrale du Togo. Au total, 52 échantillons d'eau ont été analysés suivant les normes AFNOR. L'analyse en composantes principales (ACP) a permis de faire ressortir les corrélations entre les différents paramètres physico-chimiques (16) et de comprendre les processus pouvant être à l'origine de la minéralisation. Les cartes de distribution de la conductivité et des nitrates ont été ensuite réalisées. Les travaux révèlent que du fait de la prédominance des ions bicarbonate, calcium et magnésium, les eaux sont pour la plupart bicarbonatées calciques et magnésiennes. Les eaux sont moyennement minéralisées et de qualité acceptable. Cependant, on note que quelques échantillons présentent des teneurs de paramètres physico-chimiques supérieures aux directives de l'OMS et sont contigus aux eaux respectant ces normes traduisant ainsi le caractère discontinu et hétérogène de l'aquifère du socle.

(C) 2015 International Formulae Group. All rights reserved.

Mots clés : Région centrale, Togo, faciès hydrochimiques, pollution, eaux souterraines, socle.

\section{Physical chemistry and water quality of rural water supply drilling in Central region in Togo}

\begin{abstract}
This study focused on the physicochemical characterization of rural water supply drilling made on base aquifers of central region of Togo. In total, 52 samples of water were analyzed according to the AFNOR Standards. The principal component analysis (PCA) allowed to highlight correlations between different physico-chemical parameters (16) and to understand the processes that can explain source of the mineralization. Conductivity distribution and nitrate maps were then performed. The work reveals that due to the predominance of bicarbonate calcium and magnesium ions, water is mostly calcic and magnesian bicarbonated. Water is moderately mineralized with acceptable quality. However, we note that some samples exhibit physicochemical parameters levels higher than those of WHO guidelines and are contiguous to samples up to standard showing discontinuous and heterogeneous nature of base aquifer.

(c) 2015 International Formulae Group. All rights reserved.
\end{abstract}

Keywords: Central Region, Togo, hydrochemical facies, pollution, groundwater, base. 


\section{INTRODUCTION}

$\mathrm{Au}$ Togo, les eaux souterraines constituent une part importante du patrimoine hydraulique du pays et l'accès à l'eau potable des populations constitue l'une des préoccupations majeures de l'Etat. Depuis des décennies, le pays s'est doté d'infrastructures hydrauliques adéquates pour garantir une alimentation en eau potable des localités rurales. Dans le centre du pays, excepté la ville de Sokodé et les localités environnantes alimentées par les eaux de surface, les chefslieux de préfectures sont alimentés par l'eau souterraine mise à leur disposition par la Togolaise des Eaux. Dans les milieux ruraux, l'approvisionnement en eau des populations se fait à partir des puits traditionnels et par des forages équipés de pompes à motricité humaine mis en place par le Ministère en charge de l'eau et gérés par les Comités de Développement Villageois (CDV). Le taux de desserte en eau potable de la région en 2007 est estimé à $41 \%$ (DGEA, 2009). Cependant, face à une croissance démographique importante et la vétusté de ces ouvrages d'hydraulique villageoise, les besoins en eau des populations se sont de plus en plus accrus et les ouvrages réalisés sont devenus insuffisants, ce qui entraine des pénuries d'eau dans la région. Vu le caractère discontinu des aquifères, la région est également confrontée à des problèmes d'eau qui sont récurrents dans les zones de socle. Dans cette zone, les nappes de fractures sont peu productives. Les débits de forages sont faibles et varient entre 0,5 $\mathrm{m}^{3} / \mathrm{h}$ à un peu plus de $10 \mathrm{~m}^{3} / \mathrm{h}$ (PNUD, 1982). L'approvisionnement en eau potable des populations de la région provient essentiellement des aquifères discontinus, il est indispensable de faire une caractérisation physico-chimique de ces ressources en eau et prendre connaissance de leur qualité. Les eaux souterraines, souvent protégées géologiquement, sont exposées à des pollutions agricole, industrielle ou urbaine. La consommation d'une eau contaminée par les microorganismes est à l'origine des épidémies. De même, la pollution azotée des eaux de consommation peut engendrer la méthémoglobinémie chez les nourrissons et des maladies cancérigènes chez les adultes (WHO, 2006). Vu la multiplication des forages d'hydraulique villageoise, le présent travail s'intéresse à l'étude de la qualité de même que la physico-chimie des eaux qu'ils captent.

\section{MATERIEL ET METHODES Zone d'étude}

La région centrale est une des régions économiques du Togo (Figure 1). Elle comporte 04 préfectures que sont Tchaoudjo, Sotouboua, Blitta et Tchamba. Selon le dernier recensement, elle compte 617871 habitants (DGSCN, 2011) soit 10\% de la population totale sur une superficie de 13470 $\mathrm{km}^{2}$. Limitée par les régions de la Kara et des Plateaux, la République du Bénin et la République du Ghana (Figure 1), la région centrale se situe entre le parallèle $8^{\circ}$ et le parallèle $9^{\circ} 15^{\prime}$ de latitude Nord et les méridiens $0^{\circ} 15^{\prime}$ et $01^{\circ} 35^{\prime}$ de longitude Est (PNUD-DTCD, 1981).

Elle jouit d'un climat tropical humide caractérisé par deux saisons distinctes, l'une pluvieuse et l'autre sèche de durée presqu'égale (Gù-Konu et al., 1981). La pluviométrie annuelle de la région est comprise entre $1200 \mathrm{~mm}$ et $1500 \mathrm{~mm}$ pour un nombre de jour variant entre 100 et 120 jours. La température varie entre $20^{\circ} \mathrm{C}$ et $32{ }^{\circ} \mathrm{C}$, avec des minima pendant l'harmattan, et les maxima entre les mois de février-mars. La Figure 2 montre que les mois de juin, juillet, août et septembre sont les mois où les précipitations sont supérieures à l'évapotranspiration (ETP) potentielle correspondent à la période de recharge des nappes par les précipitations.

L'agriculture est la principale activité économique de la zone. Cependant, on note une dégradation des surfaces cultivables. Les 
projets de développement s'attellent donc à la restauration des terres dégradées par leur fertilisation permanente (PNUD-DTCD, 1981).

Sur le plan géologique, la région centrale appartient à la zone des Dahoméyides et s'étend entre la zone externe, la zone de suture et la zone interne (Tairou, 2006). La zone externe comprend l'unité structurale de Buem renfermant des formations sédimentaires faiblement métamorphiques, l'unité structurale de l'Atacora avec ses sousunités schisteuses et quartzitiques et les unités de socle interne. L'ensemble de l'unité de l'Atacora encore appelée Monts Togo est affecté par une tectonique cassante. Les différentes unités structurales de la zone sont chevauchantes les unes sur les autres suivant une flèche de direction Ouest (Godonou et al., 1986). La faible épaisseur de l'altération, la faible perméabilité des produits altérés et les fortes amplitudes piézométriques font que, les puits qui tarissent pendant la saison sèche, ne sont pas l'ouvrage de captage adapté. Le forage s'avère comme le moyen de captage permettant d'exploiter de façon optimale les aquifères du socle avec un taux de réussite moyen de $70 \%$ et des débits supérieurs à 700 1/h (PNUD, 1982)

Sur le plan hydrologique, la région centrale se partage en deux grands bassins hydrographiques séparés par des chaînes de montagnes successives en écharpe qui évoluent du Sud-Ouest au Nord-Est avec des points culminants au Centre Ouest et au Centre Nord. Ce sont le bassin du Mono à l'Est et celui de la Volta à l'Ouest. Les ressources en eau renouvelables sont estimées à 4,38 milliards de $\mathrm{m}^{3} / \mathrm{an}$, dont 1,96 milliards de mètres cubes de ressources en eau souterraine et 2,42 milliards de mètres cubes de ressources en eau de surface. En dehors du barrage d'Aléhéridè qui procure un volume annuel de $476139 \mathrm{~m} 3$ (TDE-SDP, 2010) pour l'alimentation en eau potable de la ville de Sokodé et environs, l'alimentation des autres centres urbains et des localités rurales est faite essentiellement à partir des eaux souterraines. Les débits des forages rencontrés dans la zone sont faibles et varient entre $0,5 \mathrm{~m}^{3} / \mathrm{h}$ à un peu plus de $10 \mathrm{~m}^{3} / \mathrm{h}$ (PNUD, 1982 ; DGEA, 2009). La série de Buem apparaît comme une excellente réserve d'eau souterraine dans la région.

\section{Méthodologie}

Cette étude a été effectuée sur 52 échantillons d'eau de forages captant le socle cristallin dans la région centrale du Togo. La campagne de mesures, a été réalisée en mars 2005 correspondant à la période des basses eaux. La Figure 1 indique la position géographique des sites d'échantillonnage.

Avant tout prélèvement, les forages ont été purgés afin d'avoir un représentatif de l'eau de la nappe. Nous avons laissé écouler l'eau du forage pendant quelques minutes. Pour chaque point d'eau, nous avons prélevé deux échantillons dans des bouteilles en polyéthylène de 1,5 1. Les échantillons ont été transportés dans des glacières au Laboratoire de Chimie des Eaux de 1'Université de Lomé pour être analysés. Pour le dosage des cations, nous avons procédé à l'acidification des échantillons avec de l'acide nitrique. Cette opération est destinée à maintenir en solution tous les éléments susceptibles d'être précipités en l'occurrence le fer et le manganèse. Le matériel et les méthodes (AFNOR, 1986) utilisés pour l'analyse physico-chimique sont consignés dans le Tableau 1. La validité des résultats des analyses hydrochimiques a été réalisée en utilisant la méthode de la balance ionique à l'aide du logiciel «Diagrammes ». L'erreur commise sur la balance ionique pour tous les résultats est inférieure à $5 \%$. Sur le terrain, nous avons procédé aux mesures de paramètres physiques tels le $\mathrm{pH}$ et la conductivité électrique à l'aide des sondes multiparamètres de marque WTW 330. Un GPS de type Garmin a permis de 
géoréférencer les différents points d'eau échantillonnés.

A l'aide du diagramme de Piper, nous avons procédé à l'identification des différentes familles d'eau en utilisant le logiciel «Diagrammes » (Simler, 2006). Ce diagramme rend compte de la proportion des ions majeurs dans une eau (Atteia, 2005 ; Banton et Bangoy, 1997). L'analyse en composante principale (ACP) a permis de faire ressortir les corrélations entre les différents paramètres physico-chimiques et de comprendre les processus pouvant être à l'origine de la minéralisation. Le programme utilisé à cet effet est Xlstat (Famhy, 2006). Les logiciels MapInfo et Surfer nous ont servi dans l'établissement des différentes cartes de distribution. Etant donné la discontinuité des aquifères de socle, ces cartes de distribution n’ont pas été représentées par les cartes en courbe isovaleurs, mais par des points dont la couleur reflète la plage de concentration de l'élément dans le puits échantillonné. L'analyse de la qualité physico-chimique des eaux a été faite par rapport aux normes OMS (WHO, 2006).

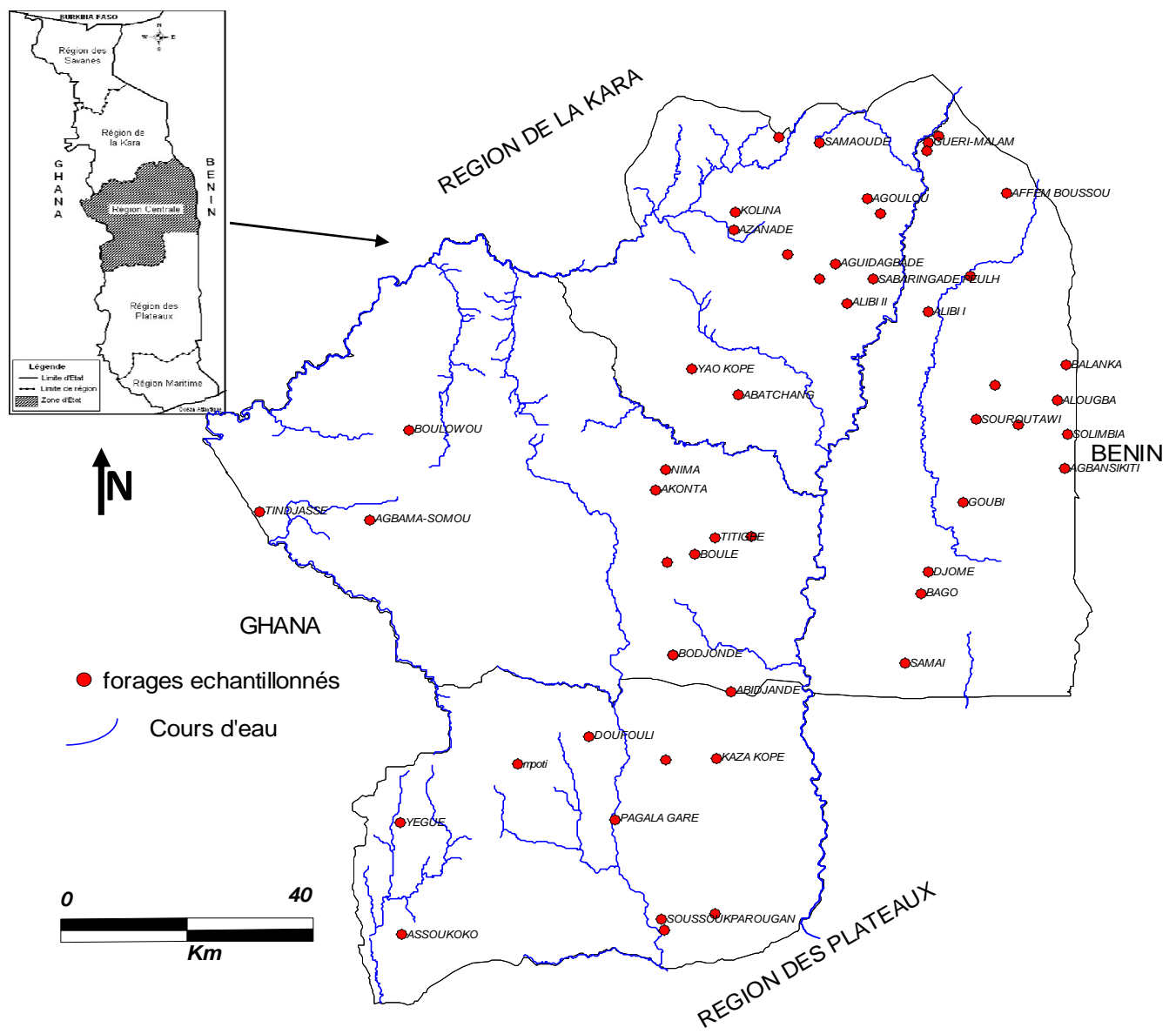

Figure 1 : Localisation géographique des sites. 


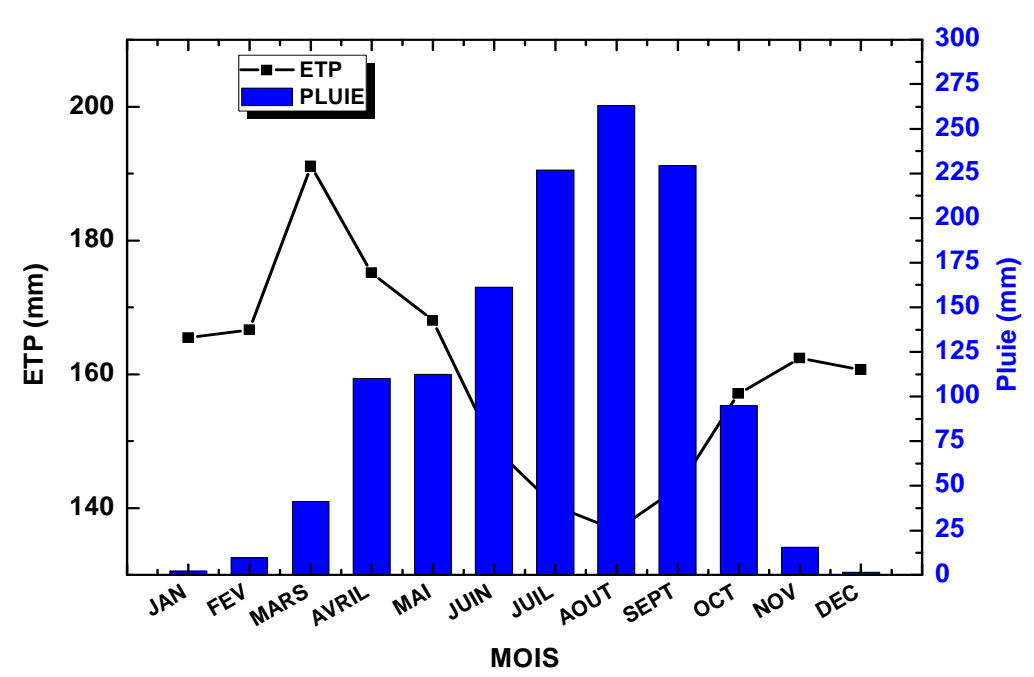

Figure 2 : Variation mensuelle de la précipitation et de l'évapotranspiration.

\section{RESULTATS}

$\mathrm{Vu}$ le grand nombre des sites échantillonnés (52) et celui des paramètres considérés (16), nous avons jugé préférable de présenter les résultats des analyses physicochimiques sous forme statistique (Tableau 2). Pour chaque paramètre physico-chimique, nous avons calculé le maximum, le minimum, l'écart type et la moyenne. A ces différents paramètres, nous avons ajouté les profondeurs des ouvrages pour les besoins d'interprétation.

A part le $\mathrm{pH}$, les ions ammonium $\left(\mathrm{NH}_{4}^{+}\right)$, les ions nitrite $\left(\mathrm{NO}_{2}^{-}\right)$, les ions manganèse $\left(\mathrm{Mn}^{2+}\right)$ et le fer total $\left(\mathrm{Fe}_{\mathrm{T}}\right)$ qui présentent des écarts types très faibles (inférieurs à 2), les autres paramètres physicochimiques présentent des écarts-types élevés traduisant leur dispersion autour de leur moyenne.

Le $\mathrm{pH}$ des eaux récoltées varient entre 5,25 et 7,82 respectivement à Boulohou et Soussoukparogan. $60 \%$ des échantillons récoltés ont un $\mathrm{pH}$ franchement acide $(<7)$. Le reste des échantillons présentent des $\mathrm{pHs}$ proches de la neutralité. L'acidité de ces eaux résulterait de la présence de dioxyde de carbone dissous $\left(\mathrm{CO}_{2}\right.$ libre $)$.
Les conductivités électriques varient entre 40 et $1600 \mu \mathrm{S} / \mathrm{cm}$ avec trois localités présentant des valeurs qui excèdent la norme OMS de $1000 \mathrm{mg} / \mathrm{l}$. Les éléments qui influencent cette conductivité sont le calcium, le magnésium, les chlorures, les bicarbonates, le sodium, le potassium, les sulfates et les éléments azotés tels que les nitrates et les nitrites (Tableau 3). La Figure 3 représente la carte de distribution des conductivités sur un fond géologique de la région. Les eaux dominantes sont celles de minéralisation peu accentuée à moyenne. Cette carte montre en outre que 25 forages soit $48 \%$ des sites échantillonnés présentent des conductivités comprises entre 200 et $400 \mu \mathrm{S} / \mathrm{cm}$. Ces eaux sont à minéralisation peu accentuée. Les eaux de faible minéralisation sont rencontrées dans des formations quartzitiques. Trois échantillons classés dans les eaux à minéralisation excessives sont localisé au Nord Est de la région dans les villages de Balanka, Afem et Koussountou dans la préfecture de Tchamba. La répartition spatiale de la conductivité montre que celle-ci est indépendante de la lithologie; ainsi une même formation géologique, présente une diversité 
de conductivité. La carte de distribution des conductivités montre que celles-ci ne sont pas intimement liées aux différentes formations géologiques de la région.

La valeur minimale de la turbidité est 0,17 NTU tandis que la valeur maximale est de 430 NTU. Pour une désinfection efficace, l'OMS recommande des turbidités inférieures à 5 NTU (ACF, 2006). 17 forages ont une valeur de ce paramètre supérieure à 5 NTU. Les turbidités élevées remarquées essentiellement à l'Ouest sont contiguës aux turbidités basses.

La dureté des eaux souterraines de la région varie entre $0,8{ }^{\circ} \mathrm{f}$ et $72,6^{\circ} \mathrm{f}$, avec une moyenne de $17,24^{\circ} \mathrm{f}$. Les valeurs de la dureté permettent de classer les eaux de la région dans la plage allant des eaux douces aux eaux très dures. Les eaux très dures dont le titre hydrotimétrique est supérieur à $50{ }^{\circ} \mathrm{f}$ sont rencontrées au niveau de trois villages (Balanka, Affem Boussou, Koussountou). Ces localités présentent également des teneurs les plus élevées en calcium et en salinité.

Les eaux de la région sont caractérisées par une prédominance des ions bicarbonates $(200 \mathrm{mg} / \mathrm{l})$ sur les ions chlorures $(20 \mathrm{mg} / \mathrm{l})$ et les sulfates $(10 \mathrm{mg} / \mathrm{l})$. Les éléments azotés dosés dans les eaux sont les nitrates, les nitrites et l'ammonium. La concentration moyenne obtenue en ion ammonium dans les eaux de la région est de $0,07 \mathrm{mg} / \mathrm{l}$ avec une valeur maximale de $0,6 \mathrm{mg} / \mathrm{l}$. C'est le paramètre qui présente la plus faible dispersion $(0,07)$. Quant aux formes oxydées (nitrites et nitrates), les analyses indiquent une concentration moyenne en nitrites de l'ordre de $0,17 \mathrm{mg} / \mathrm{l}$. Des teneurs en nitrites comprises entre 1,03 et $1,94 \mathrm{mg} / \mathrm{l}$ ont été retrouvées dans les forages de Soussoukpa, Akonta et Koulan. Toutes les eaux échantillonnées sont ainsi conformes à la norme OMS (3 mg/l). Les concentrations en nitrates des eaux oscillent entre 0,5 et 263 $\mathrm{mg} / \mathrm{l}$. Des trois composés minéraux azotés étudiés $\mathrm{NH}_{4}^{+}, \mathrm{NO}_{2}^{-}$et $\mathrm{NO}_{3}^{-}$, seul les $\mathrm{NO}_{3}^{-}$ présentent des valeurs qui s'écartent de la norme OMS. La Figure 4 représente la carte de distribution des nitrates dans la région par rapport à la norme OMS qui est de $50 \mathrm{mg} / \mathrm{l}$ (WHO, 2006). Douze (12) forages ont des concentrations en nitrates supérieures à cette norme. Ces forages sont situés au Nord Est de la région dans un secteur à cheval sur les préfectures de Tchamba et de Tchaoudjo d'un part et dans la partie sud de la préfecture de Blitta d'autre part. La distribution des ions nitrates est similaire à celle des conductivités et semble montrer que ces dernières influencent la conductivité. Ceci est confirmé par le coefficient de corrélation entre les deux éléments qui est de 8,5 (Tableau 3).

Les ions calcium et sodium constituent les cations les plus dominants avec des teneurs moyennes respectives de 41,6 et $21,8 \mathrm{mg} / \mathrm{l}$. Le cation majeur le moins représenté est le potassium avec une teneur moyenne de 9,67 $\mathrm{mg} / \mathrm{l}$. Seuls le $\mathrm{K}^{+}$et le $\mathrm{Ca}^{2+}$ présente des teneurs qui excèdent leurs concentrations maximales admises, CMA (12 et $200 \mathrm{mg} / \mathrm{l})$. Que ce soit pour les cations que pour les anions, les importants écarts types enregistrés au niveau de ces paramètres physicochimiques montrent que les eaux de la région présentent dans leur ensemble une hétérogénéité chimique.

Concernant les métaux lourds, l'analyse des eaux souterraines de la région centrale a révélé la présence de fer et du manganèse. Le fer est le principal métal dans les eaux avec une teneur moyenne de l'ordre de $0,86 \mathrm{mg} / \mathrm{l}$ et des teneurs extrêmes de 0,05 et $1,47 \mathrm{mg} / \mathrm{l}$. Vingt-quatre forages (24) soit 46 $\%$ des échantillons ont des teneurs en fer supérieures à la concentration maximale admissible de l'OMS (0,3 $\mathrm{mg} / \mathrm{l})$. Le manganèse est présent dans les eaux à raison de $0,49 \mathrm{mg} / \mathrm{l}$ en moyenne. Vingt-trois forages (23) ont des teneurs en manganèse qui dépasse la concentration limite recommandée $(0,4$ $\mathrm{mg} / \mathrm{l})$. Les concentrations en chlorure $\left(\mathrm{Cl}^{-}\right)$et 
sulfate $\mathrm{SO}_{4}^{2-}$ respectent les normes OMS de $250 \mathrm{mg} / \mathrm{l} \mathrm{et} 400 \mathrm{mg} / \mathrm{l}$ respectivement.

Le Tableau 3 résume les résultats obtenus. Les coefficients en gras sont ceux qui présentent une corrélation significative. On note une corrélation très significative entre la conductivité $\mathrm{CE}$ et les bicarbonates $\mathrm{HCO}_{3}^{-}$ $(\mathrm{r}=0,73)$, les nitrates $\mathrm{NO}_{3}^{-}(\mathrm{r}=0,85)$, les nitrites $\mathrm{NO}_{2}^{-}(\mathrm{r}=0,5)$, les ions calcium $\mathrm{Ca}^{2+}$ $(\mathrm{r}=0,89)$, les ions magnésium $\mathrm{Mg}^{2+}$ $(\mathrm{r}=0,85)$, le sodium $\mathrm{Na}^{+}(0,54)$, les ions potassium $\mathrm{K}^{+}(0,7)$, les ions $\mathrm{Cl}^{-}(\mathrm{r}=0,86)$, les ions $\mathrm{SO}_{4}^{2-}(0,65)$. Le pH n'est corrélé qu'avec les hydrogénocarbonates $(r=0,58)$. Ces derniers sont à leur tour corrélés avec le calcium $(r=0,69)$ et le magnésium $(r=0,80)$. La turbidité est bien corrélée avec le fer $(r=0,75)$. Une corrélation significative existe également entre les nitrates, qui sont des éléments généralement issus des activités anthropiques, et le calcium, le magnésium, le sodium, le potassium et dans une moindre mesure les sulfates. Il en est de même entre les nitrites et le sodium et dans une moindre mesure avec les chlorures $(r=0,45)$.

Il n'existe aucune corrélation entre les chlorures et le sodium, de même que la profondeur avec tous les paramètres.

Les familles hydrochimiques des échantillons analysés sont représentées sur la Figure 5. On peut identifier 04 familles à savoir une famille bicarbonatée calcique et magnésienne pour $84,61 \%$ (44 forages), une famille chlorurée calcique et magnésienne pour $13,46 \%$ des échantillons (06 forages). En plus de ces deux grandes familles, on note la présence d'une famille chlorurée sodique potassique (Koulan) et d'une famille chlorurée calcique (Koussountou).

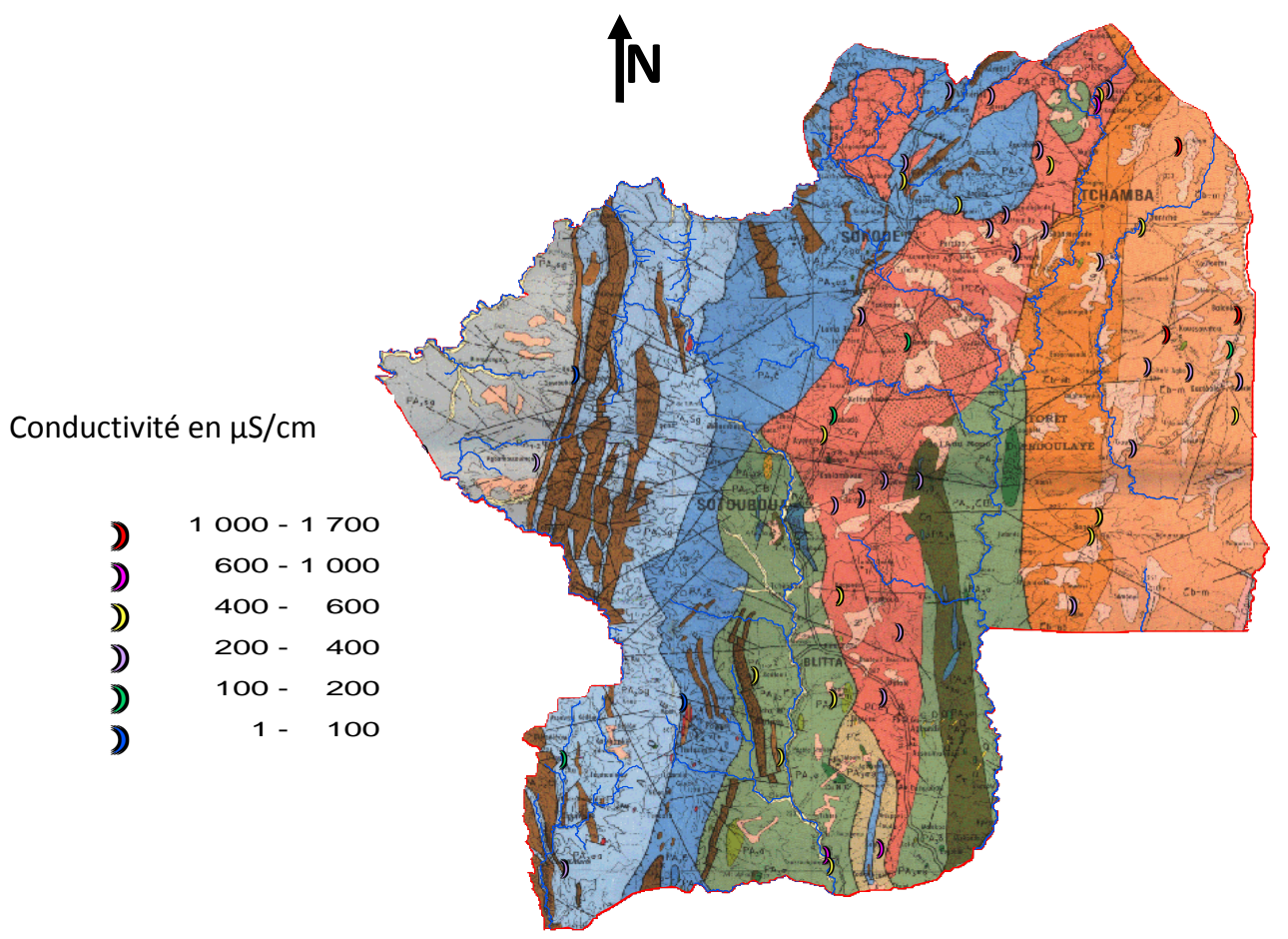

Figure 3 : Carte de distribution de la conductivité. 


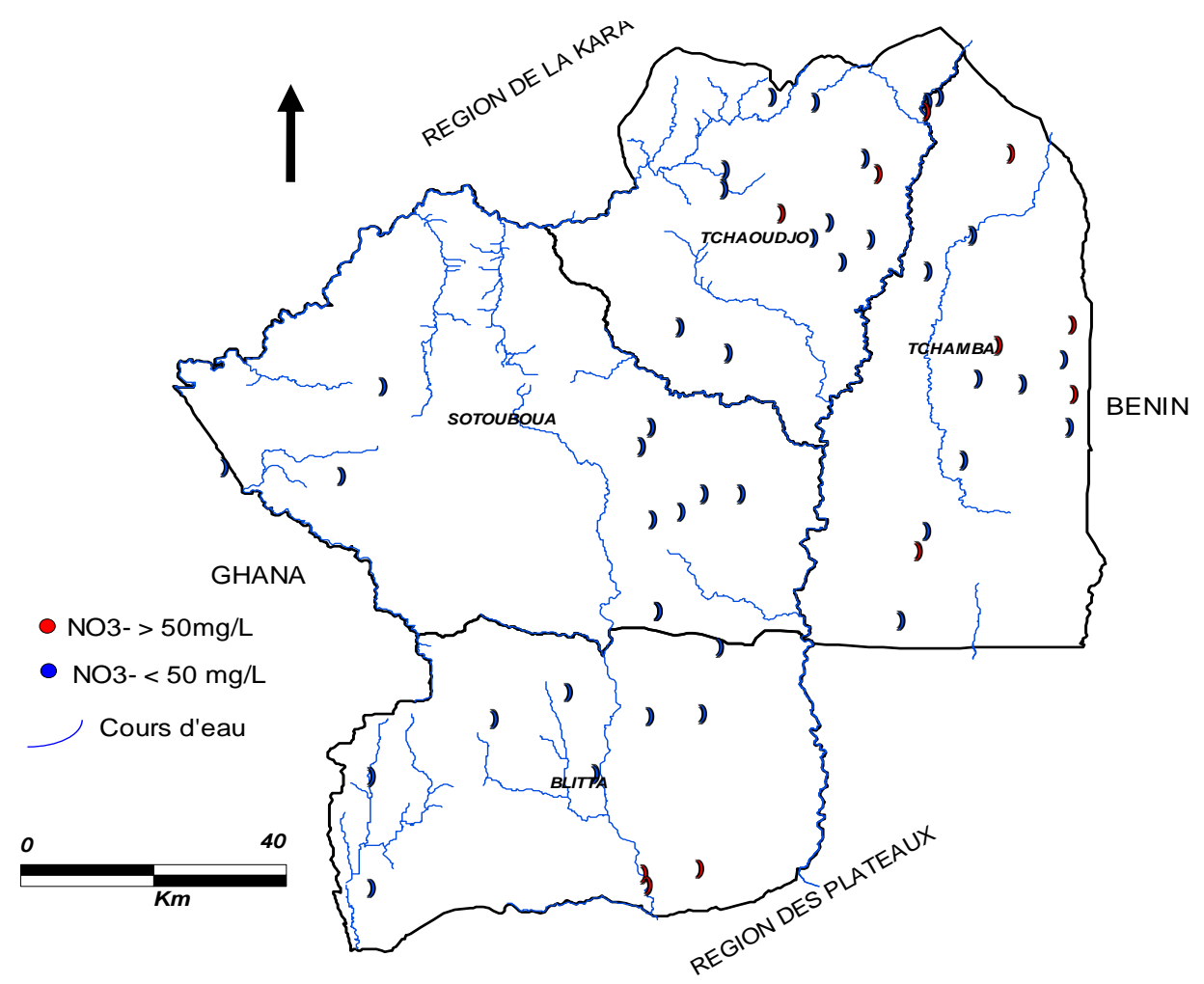

Figure 4 : Carte de distribution des nitrates.

$\triangle$

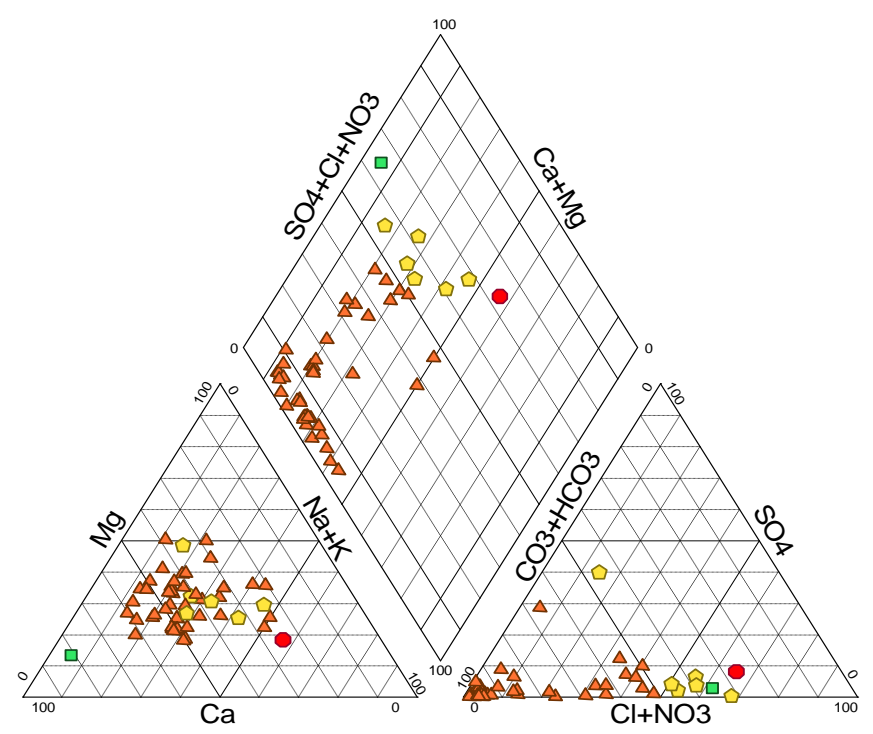

Figure 5 : Familles hydrochimiques des eaux 
Tableau 1 : Matériel et méthodes utilisés pour le dosage des paramètres physico chimiques.

\begin{tabular}{|c|c|c|c|c|}
\hline Paramètres & Méthodes & $\begin{array}{l}\text { Norme } \\
\text { AFNOR }\end{array}$ & $\begin{array}{l}\text { Précision de } \\
\text { la méthode }\end{array}$ & Matériel \\
\hline $\mathrm{pH}$ & Electrométrie & NFT 90-008 & \pm 0.01 & pH- mètre WTW pH 330i \\
\hline Turbidité & Néphélométrie & NFT 90-33 & $\pm 0.01 \mathrm{NTU}$ & $\begin{array}{l}\text { Turbidimètre DRT100B } \\
\text { model } 20012\end{array}$ \\
\hline (CE) & Conductimétrie & NFT 90-031 & $\pm 0.5 \%$ & $\begin{array}{c}\text { Conductimètre WTW } \\
\text { Cond 330i }\end{array}$ \\
\hline $\mathrm{Ca}^{2+}$ & Complexométrie à l'EDTA & NFT 90-016 & $\pm 0.5 \mathrm{mg} / \mathrm{l}$ & - \\
\hline $\mathrm{Mg}^{2+}$ & Complexométrie à l'EDTA & NFT 90-016 & $\pm 0.24 \mathrm{mg} / \mathrm{l}$ & - \\
\hline $\mathrm{Na}^{+}$ & $\begin{array}{l}\text { Spectrophotométrie } \\
\text { d'absorption atomique }\end{array}$ & NFT 90-20 & $\pm 0.04 \mathrm{mg} / \mathrm{l}$ & $\begin{array}{c}\text { Spectrophotomètre } \\
\text { Perkin Elmer model } 2380\end{array}$ \\
\hline $\mathrm{K}^{+}$ & $\begin{array}{l}\text { Spectrophotométrie } \\
\text { d'absorption atomique }\end{array}$ & NFT 90-20 & $\pm 0.02 \mathrm{mg} / \mathrm{l}$ & $\begin{array}{c}\text { Spectrophotomètre } \\
\text { Perkin Elmer model } 2380\end{array}$ \\
\hline $\mathbf{N H}_{4}^{+}$ & $\begin{array}{c}\text { Spectrophotométrie } \\
\text { d'absorption moléculaire }\end{array}$ & NFT 90-015 & 1 à $2 \%$ & $\begin{array}{l}\text { Spectrophotomètre } \\
\text { Digitron Elvi } 675\end{array}$ \\
\hline $\mathrm{Fe}_{\mathrm{T}}$ & $\begin{array}{c}\text { Spectrophotométrie } \\
\text { d'absorption moléculaire }\end{array}$ & NFT 90-017 & 1 à $2 \%$ & $\begin{array}{l}\text { Spectrophotomètre } \\
\text { Digitron Elvi } 675\end{array}$ \\
\hline $\mathrm{Mn}^{2+}$ & $\begin{array}{c}\text { Spectrophotométrie } \\
\text { d'absorption moléculaire }\end{array}$ & NFT 90-024 & 1 à $2 \%$ & $\begin{array}{l}\text { Spectrophotomètre } \\
\text { Digitron Elvi } 675\end{array}$ \\
\hline $\mathrm{Cl}^{-}$ & Argentimétrie & NFT 90-014 & $\pm 0.5 \mathrm{mg} / \mathrm{l}$ & - \\
\hline $\mathrm{HCO}_{3}^{-}$ & Acidimétrie & NFT 90-036 & $\pm 0.25 \mathrm{mg} / \mathrm{l}$ & $\begin{array}{l}\text { Spectrophotomètre } \\
\text { Digitron Elvi } 675\end{array}$ \\
\hline $\mathrm{SO}_{4}{ }^{2-}$ & Néphélométrie & NFT 90-009 & 1 à $2 \%$ & $\begin{array}{l}\text { Spectrophotomètre } \\
\text { Digitron Elvi } 675\end{array}$ \\
\hline $\mathrm{NO}_{3}^{-}$ & $\begin{array}{c}\text { Spectrophotométrie } \\
\text { d'absorption moléculaire }\end{array}$ & NFT 90-012 & 1 à $2 \%$ & $\begin{array}{l}\text { Spectrophotomètre } \\
\text { Digitron Elvi } 675\end{array}$ \\
\hline $\mathrm{NO}_{2}^{-}$ & $\begin{array}{c}\text { Spectrophotométrie } \\
\text { d'absorption moléculaire }\end{array}$ & NFT 90-013 & 1 à $2 \%$ & $\begin{array}{l}\text { Spectrophotomètre } \\
\text { Digitron Elvi } 675\end{array}$ \\
\hline
\end{tabular}

Tableau 2 : Résultats statistiques des paramètres physico-chimiques $(\mathrm{n}=52)$.

\begin{tabular}{lcccc}
\hline Paramètres & Minimum & Maximum & Moyenne & Ecart type \\
\hline $\mathrm{pH}$ & 5,25 & 7,82 & 6,76 & 0,52 \\
$\mathrm{CE}$ & 40,00 & 1600,00 & 439,45 & 321,85 \\
Turb. & 0,17 & 430,00 & 25,46 & 78,36 \\
$\mathrm{TH}$ & 0,80 & 72,60 & 17,24 & 13,36 \\
$\mathrm{Ca}^{2+}$ & 1,60 & 248,00 & 41,60 & 38,20 \\
$\mathrm{Mg}^{2+}$ & 1,00 & 64,80 & 16,42 & 12,20 \\
$\mathrm{Na}^{+}$ & 2,40 & 99,30 & 21,80 & 18,31 \\
$\mathrm{~K}^{+}$ & 0,22 & 140,40 & 9,67 & 20,48 \\
$\mathbf{F e}_{\mathbf{T}}$ & 0,05 & 7,60 & 0,86 & 1,47 \\
\hline
\end{tabular}


A. A. DOUGNA et al. / Int. J. Biol. Chem. Sci. 9(4): 2249-2262, 2015

\begin{tabular}{lcccc}
\hline $\mathbf{M n}^{2+}$ & 0,05 & 4,47 & 0,49 & 0,74 \\
$\mathbf{N H}_{4}^{+}$ & 0,05 & 0,61 & 0,07 & 0,08 \\
$\mathbf{H C O}_{3}^{-}$ & 12,20 & 579,50 & 200,40 & 104,07 \\
$\mathbf{N O}_{2}^{-}$ & 0,025 & 1,94 & 0,17 & 0,37 \\
$\mathbf{C l}^{-}$ & 1,00 & 190,21 & 20,96 & 32,54 \\
$\mathbf{S O}_{4}^{2-}$ & 0,23 & 72,50 & 9,98 & 16,78 \\
$\mathbf{N O}_{3}^{-}$ & 0,50 & 263,00 & 39,40 & 73,31 \\
Prof & 36,15 & 85,85 & 56,56 & 12,31 \\
\hline
\end{tabular}

Tableau 3 : Coefficients de corrélation (r) entre les différents paramètres

\begin{tabular}{|c|c|c|c|c|c|c|c|c|c|c|c|c|c|c|c|c|}
\hline Variables & $\mathrm{CE}$ & $\mathrm{pH}$ & Turb. & $\mathrm{HCO}_{3}^{-}$ & $\mathrm{NH}_{4}^{+}$ & $\mathrm{NO}_{3}^{-}$ & $\mathrm{NO}_{2}^{-}$ & $\mathrm{Ca}^{2+}$ & $\mathrm{Mg}^{2+}$ & $\mathrm{Na}^{+}$ & $\mathrm{K}^{+}$ & $\mathrm{Fe}_{\mathrm{T}}$ & $\mathrm{Mn}^{2+}$ & $\mathrm{Cl}^{-}$ & $\mathrm{SO}_{4}^{2-}$ & Prof \\
\hline $\mathrm{CE}$ & 1 & & & & & & & & & & & & & & & \\
\hline $\mathrm{pH}$ & 0,41 & 1 & & & & & & & & & & & & & & \\
\hline Turb. & $-0,13$ & $-0,35$ & 1 & & & & & & & & & & & & & \\
\hline $\mathrm{HCO}_{3}^{-}$ & $\mathbf{0 , 7 3}$ & $\mathbf{0 , 5 8}$ & $-0,21$ & 1 & & & & & & & & & & & & \\
\hline $\mathrm{NH}_{4}^{+}$ & $-0,04$ & $-0,22$ & 0,68 & $-0,12$ & 1 & & & & & & & & & & & \\
\hline $\mathrm{NO}_{3}^{-}$ & 0,85 & 0,13 & $-0,06$ & 0,35 & $-0,06$ & 1 & & & & & & & & & & \\
\hline $\mathrm{NO}_{2}^{-}$ & 0,50 & 0,29 & 0,01 & 0,20 & 0,08 & $\mathbf{0 , 5 0}$ & 1 & & & & & & & & & \\
\hline $\mathrm{Ca}^{2+}$ & 0,89 & 0,33 & $-0,13$ & 0,69 & $-0,06$ & 0,65 & 0,26 & 1 & & & & & & & & \\
\hline $\mathrm{Mg}^{2+}$ & 0,83 & 0,39 & $-0,12$ & 0,80 & $-0,03$ & 0,65 & 0,32 & 0,63 & 1 & & & & & & & \\
\hline $\mathrm{Na}^{+}$ & 0,54 & 0,46 & $-0,13$ & 0,26 & $-0,05$ & 0,62 & 0,64 & 0,20 & 0,39 & 1 & & & & & & \\
\hline $\mathrm{K}^{+}$ & 0,70 & 0,14 & $-0,07$ & 0,67 & 0,01 & 0,58 & 0,33 & 0,48 & 0,75 & 0,29 & 1 & & & & & \\
\hline $\mathrm{Fe}_{\mathrm{T}}$ & $-0,28$ & $-0,44$ & 0,75 & $-0,28$ & 0,61 & $-0,24$ & $-0,08$ & $-0,24$ & $-0,26$ & $-0,26$ & $-0,14$ & 1 & & & & \\
\hline $\mathrm{Mn}^{2+}$ & 0,38 & 0,16 & $-0,03$ & 0,10 & $-0,10$ & 0,30 & 0,06 & $\mathbf{0 , 5 4}$ & 0,08 & 0,03 & $-0,07$ & $-0,09$ & 1 & & & \\
\hline $\mathrm{Cl}^{-}$ & 0,86 & 0,24 & $-0,09$ & 0,42 & $-0,02$ & 0,78 & 0,45 & 0,86 & 0,59 & 0,39 & 0,40 & $-0,23$ & 0,60 & 1 & & \\
\hline $\mathrm{SO}_{4}^{2-}$ & 0,65 & 0,18 & 0,17 & 0,48 & 0,36 & $\mathbf{0 , 5 1}$ & 0,35 & 0,51 & 0,62 & 0,37 & 0,62 & 0,06 & 0,00 & 0,44 & 1 & \\
\hline Prof & $-0,02$ & $-0,19$ & 0,13 & 0,16 & $-0,08$ & $-0,10$ & 0,05 & $-0,05$ & 0,11 & $-0,16$ & 0,24 & 0,14 & $-0,23$ & $-0,16$ & 0,02 & 1 \\
\hline
\end{tabular}

\section{DISCUSSION}

La connaissance de la nature lithologique de la roche aquifère est importante pour mieux comprendre la géochimie de l'aquifère (Boubakar Hassane, 2010). Les écarts-types très élevés révèlent que les paramètres sont très dispersés autour de leur moyenne, ce qui rend compte de la diversité tant structurale que lithologique du réservoir que constitue le socle (Belghoul, 2007 ; Vouillamoz, 2003).

Les résultats de l'analyse physicochimique présentés dans ce travail, montrent que les sulfates $(9,8 \mathrm{mg} / \mathrm{l})$ et les chlorures 
$(20,96)$ sont compatibles avec les normes des eaux d'alimentation. Le $\mathrm{pH}$ doit être compris entre 6,5 et 8,5 pour une eau potable. Le $\mathrm{pH}$ contrôle la solubilité et la vitesse de réaction des espèces métalliques impliquées dans les réactions de corrosion qui peuvent affecter la qualité microbiologique et physico-chimique des eaux. Certains forages (4 soit 7,8\% du total) ont un $\mathrm{pH}<6$ et présentent des indices d'agressivité les plus élevés (valeurs non présentées) qui peuvent entraîner la corrosion des tuyauteries métalliques (WHO, 2006). Dans notre région, il est essentiellement acide au Nord-Ouest et au Sud-Ouest dans les formations schisteuses. Un constat similaire a été fait pour les eaux de formations géologiques du socle de Niamey et a été relié aux formations géologiques à caractère acide tels les grès et les schistes. Les ions bicarbonates $\left(\mathrm{HCO}_{3}^{-}\right)$présente le plus grand écart-type par rapport aux autres anions majeurs. Ces résultats sont en accord avec ceux obtenus par Boubakar Hassane en 2010. Les eaux dont la dureté est supérieure à $20^{\circ} \mathrm{f}$ (11 au total) et situées à l'Est de la zone sont susceptibles d'occasionner l'entartage des appareils ménagers et l'obstruction les canalisations. Par contre, les eaux douces vont corroder les tuyauteries. Il faut préciser que cette notion de corrosion doit être évaluée également en fonction d'autres paramètres tels le $\mathrm{pH}$.

La situation des forages dans les dépressions explique les fortes teneurs en chlorure qui sont dues au phénomène d'évaporation. La corrélation est faible entre les ions chlorure $(r=0,39)$ et les ions sodium. Cependant elle est forte avec les ions nitrates $(\mathrm{r}=0,78)$ traduisant la même origine anthropique et atmosphérique puisque les formations géologiques sont exemptes de nitrate.

Les nitrates sont considérés dangereux pour la santé lorsque leurs concentrations excèdent la norme OMS de $50 \mathrm{mg} / \mathrm{l}$. Ces ions ingérés lors de la consommation de l'eau sont réduits en nitrites dans les intestins et se fixent sur l'hémoglobine, diminuant ainsi sa capacité de transfert de l'oxygène (ACF, 2006 ; WHO, 2006). Cette affection, connue sous le nom de méthémoglobinémie, touche surtout les enfants de bas âges. Il serait donc important que ces eaux soient déconseillées à la population.

La pollution nitratée des eaux souterraines étudiées serait due à l'utilisation massive des fertilisants azotés, la zone étant à forte activité agricole caractérisée surtout par la culture cotonnière. La nature sabloargileuse des sols et l'importante fracturation de la zone contribue à l'aggravation de la pollution nitratée. Plus les formations géologiques sont perméables et peu épaisses, plus les concentrations en nitrate dans la nappe sous-jacente seront élevées (Vouillamoz, 2003) car favorisées par les conditions aérobies. La variabilité spatiale des nitrates constatée entre les différents forages peut être liée entre autres à l'hétérogénéité du milieu physique : la texture de la surface, la lithologie et la fracturation. Les fortes précipitations enregistrées dans la zone et la destruction du couvert végétal contribueraient à la lixiviation rapide des nitrates vers la nappe (Atteia, 2005). Le $\mathrm{HCO}_{3}^{-}$présente le plus grand écart type par rapport aux autres anions majeurs (Boubakar Hassane, 2010). Etant en outre l'anion dominant, $\mathrm{Ca}^{2+}, \mathrm{Mg}^{2+}$, $\mathrm{K}^{+}, \mathrm{Na}^{+}$proviendraient de l'altération des minéraux silicatés à cause de la réaction s'effectuant entre l'acide carbonique et les minéraux feldspathiques en milieux aqueux et qui aboutit à la mise en solution de $\mathrm{HCO}_{3}^{-}$ (Lloyd et Heathcote, 1985). Il existe une forte corrélation entre les éléments majeurs et la conductivité électrique. Ces éléments seraient donc à l'origine de la minéralisation des eaux. Les eaux les plus minéralisées se retrouvent dans les gneiss migmatitiques (Godonou et al., 1986). Pour des considérations organoleptiques, une teneur de $600 \mathrm{mg} / \mathrm{l}$ en 
sels dissous est conseillée bien que la CMA soit de $1000 \mathrm{mg} / \mathrm{l}$. Le calcium est l'élément majeur de la dureté totale $(\mathrm{TH})$ et de la conductivité $(\mathrm{r}=0,89)$. Seul le forage de Koussountou excède la Concentration Maximale Admise de 200 mg/l (WHO, 2006). En ce qui concerne le potassium, on note des concentrations très élevées à l'Est. Cependant ce paramètre étant sous contrôle de l'homéostasie, même ces teneurs n'auraient que des effets négligeables sur la concentration dans l'organisme (WHO, 2006). Généralement dans les eaux, le potassium est le moins abondant des cations majeurs après le calcium, le magnésium et le sodium. En effet, l'argile adsorbe sélectivement le potassium de masse atomique plus élevée que le sodium de préférence à ce dernier (Banton et Bangoy, 1997). Dans la région d'étude, le calcium représente le plus abondant des cations majeurs à l'exception de certains forages où $\mathrm{K}^{+}$est dominant (10 au total). Cet écart à la normale peut s'expliquer par l'altération des minéraux silicatés riches en potassium telle la biotite (silicate d'aluminium et de potassium), la muscovite et des feldspaths alcalins (orthose, microcline) en ces points. Selon l'OMS, le fer comme le manganèse ne doivent être éliminés des eaux que pour des considérations organoleptiques et pour empêcher la prolifération des bactéries. Cependant, des travaux effectués en Chine et au Québec ont révélé des troubles neurologiques chez des enfants d'âge scolaire (Dougna, 2011). Certains forages (18) présentent des teneurs en $\mathrm{Mn}^{2+}$ qui excède 0,4 $\mathrm{mg} / \mathrm{l}$ (WHO, 2006). Ces concentrations pourraient être dues au lessivage des altérites provenant des roches métamorphiques des minéraux silicatés tels la biotite et des feldspaths alcalins (Dougna, 2011). L'influence de la géologie sur la minéralisation a été également affirmée par d'autres auteurs (Demlie et al., 2008 ; Gnazou et al., 2011).
La turbidité n'est ni liée à une formation géologique particulière, ni à la profondeur (Tableau 3). Toutefois, on note une corrélation significative de cet élément avec le fer $(r=0,75)$. On peut donc déduire que le lessivage des sols agricoles et des argiles provenant de l'altération des roches ferromagnésiennes (pyroxène, hématite, chloritoschiste, roches amphibolitiques) seraient à l'origine de la turbidité et des teneurs en fer excédant respectivement 5 NTU et $0,3 \mathrm{mg} / \mathrm{l}$ (WHO, 2006). Cependant, les turbidités supérieures à 125 NTU pourraient être dues à un défaut de pompage avant échantillonnage.

La majorité des eaux se situe dans la famille bicarbonatée calcique et magnésienne et se retrouve dans le domaine où aucun cation n'est dominant. Des résultats similaires ont été obtenus par d'autres auteurs (Boubakar Hassane, 2010). Cette famille serait due à la diffusion du $\mathrm{CO}_{2}$ atmosphérique ou à une mise en solution du $\mathrm{CO}_{2}$ du sol dans le système ouvert que constituent les aquifères de cette région située dans une zone de fracture, L'existence de familles chlorurées s'expliquerait soit par une évaporation importante vu les températures de la région soit une altération des schistes présents dans la géologie de la localité. La famille chlorurée sodique potassique correspond à Koulang et celle chlorurée calcique à Koussountou. L'existence de ces familles s'expliquerait donc par la forte teneur dans ces forages respectivement du sodium (teneur maximale) et du calcium et chlorure (teneurs maximales). Cependant, ces familles hydrochimiques n'ont pas un caractère statique et peuvent évoluer en fonction de l'hydrodynamisme du milieu.

\section{Conclusion}

Les résultats de l'analyse physicochimique des eaux des forages d'hydraulique villageoise ont montré que les teneurs en sulfate et chlorure peuvent être considérées acceptables et ne présentent aucun danger 
pour la consommation. Les eaux de certains forages (12 au total) affichent des concentrations moyennes en nitrate supérieures aux normes de $50 \mathrm{mg} / \mathrm{l}$ selon l'organisation mondiale de la santé (OMS). Ces résultats confirment l'impact de l'intensification de l'agriculture dans une zone de socle. Il est à signaler que des forages affichent des concentrations élevées en dureté et en salinité.

Ces travaux révèlent que les eaux de forage de la région centrale présentent des faciès hydrochimiques à dominance bicarbonatés calciques et magnésiennes. La minéralisation a une origine fortement lithologique et est élevée surtout dans les formations gneissiques situées à l'Est de la zone hétérogène que constituent les formations du socle. La teneur en nitrate, est inquiétante au niveau de certains forages et a une origine anthropique et atmosphérique qui devrait être donc suivis régulièrement pour éviter un désagrément chez les consommateurs. La région centrale étant une zone à forte activité agricole, l'utilisation des engrais azotés doit être rigoureusement contrôlée afin de ne pas accentuer le problème de pollution en nitrate. Les teneurs élevées en fer et en manganèse pourraient induire un risque de développement bactérien et donc une mauvaise qualité microbiologique des eaux.

\section{REFERENCES}

ACF. 2006. Eau - Assainissement - Hygiène pour les populations à risque. Hermann, ACF.

AFNOR. 1986. Eaux : Méthodes d'Essai. AFNOR: Paris.

Demlie M, Wohnlich S, Ayenew T. 2008. Major ion hydrochemistry and environmental isotope signatures as a tool in assessing groundwater occurrence and its dynamics in a fractured volcanic aquifer system located within a heavily urbanized catchment, central Ethiopia. Journal of Hydrology 353: 175-188. doi:10.1016/j.jhydrol.2008.02.009

Atteia O. 2005. Chimie et Pollution des Eaux Souterraines. Tec \& doc :Paris.

Banton O, Bangoy LM. 1997. Hydrogéologie. Multiscience Environnementale des Eaux Souterraines. Presse de l'Université du Québec. AUPELF: Québec.

Belghoul A. 2007. Caractérisation pétrophysique et hydrodynamique du socle cristallin. Thèse de Doctorat, Université Montpellier II, p 238.

Boubakar Hassane A. 2010. Aquifères superficiels et profonds et pollution urbaine en Afrique : Cas de la communauté urbaine de Niamey (NIGER) Thèse de Doctorat, Université Abdou Moumouni de Niamey, p 198.

DGEA. 2009. Rapport de synthèse Gestion Intégrée des Ressources en Eau (GIRE) et Objectifs du Millénaire pour le Développement (OMD). UNDESA

DGSCN. 2011. Recensement Général de la Population et de l'Habitat. Ministère de la Planification du développement et de l'aménagement du territoire,Togo.

Dougna AA. 2011. Hydrogéochimie et qualité des eaux des aquifères du socle de la région centrale du Togo. Mémoire de DEA,Université de Lomé, p56.

Famhy T. 2006. www.xlstat.com. Addinsoft.

Gnazou MDT, Bawa LM, Banton O, DjaneyeBoundjou G. 2011. Hydrogeochemical characterization of the coastal Paleocene aquifer of Togo (West Africa). Afr. J. Environ. Sci. Technol. 3: 10-29. doi: 10.5897/ajest09.024

Godonou KS, Aregba A. A, Assih Edeou P. 1986. Notice explicative de la carte géologique à 1/200000. Feuille de Sokodé. Mémoire n³.DGMG/BNRM. 
Gù-Konu YE, Laclavère G, AGT. 1981. Les atlas Jeune Afrique : Atlas du Togo. Jeune Afrique.

Lloyd JW, Heathcote JA. 1985. Natural Inorganic Hydrochemistry in Relation to Groundwater. An Introduction. Clarendon Press : Oxford.

PNUD-DTCD. 1981. Atlas du développement régional du Togo. DGPD/DCNC.

PNUD. 1982. Stratégie d'Aménagement des Eaux. Ressources et Besoins en Eau. Laboratoire central d'hydraulique de France, PNUD : Paris.

Rodier J, Legube B, Merlet N. 2009. Analyse de l'Eau. DUNOD : Paris.
Simler

R. 2006.

Logiciel d'hydrochimie/Diagrammes.http//www. lha.univ-avignon.fr/logiciel.htm.

Tairou SM. 2006. La tectonique tangentielle panafricaine au Nord Togo. Thèse de doctorat. Université de Lomé, p 401.

TDE-SDP 2002. Tableau de production d'eau brute des centres et secteurs. Rapport de production.

Vouillamoz JM. 2003. La caractérisation des aquifères par une méthode non invasive: les sondages par résonance magnétique protonique. Thèse de Doctorat. Université de Paris XI, p221.

WHO. 2011. Guidelines for Drinking Water Quality. World Health Organization. 